\title{
T2SOLV: AN ENHANCED PACKAGE OF SOLVERS FOR THE TOUGH2 FAMILY OF CODES
}

\author{
George 3. Moridis and Karsten Pruess \\ Lawrence Berkeley National Laboratory \\ Earth Sciences Division, 1 Cyclotron Road, 90-1 116 \\ Berkeley, California 94720
}

\begin{abstract}
r2SOLV is an enhanced package of solvers for the TOUGH2 family of codes. T2SOLV includes all the 'reconditioned Conjugate Gradient (PCG) solvers ised in T2CG1, the current solver package, as well as ¿UBAND, a new direct solver, and DLUSTB, a PCG solver based on the BiCGSTAB method. Additionally, T2SOLV includes the D4 grid numbering scheme and two sets of preprocessors. Results From test problems indicate that LUBAND is faster, nore reliable and requires less storage than MA28, :he BiCGSTAB solver is superior to the other PCG methods in T2SOLV, and that the preprocessors improve the performance of the PCG solvers and allow the solution of previously intractable problems.
\end{abstract}

\section{INTRODUCTION}

Most of the computational work in the numerical simulations of fluid and heat flows in permeable media arises from the solution of large systems of linear equations $\mathbf{A x}=\mathbf{b}$, where $\mathbf{A}$ is a banded matrix of or$\operatorname{der} N, \mathbf{x}$ is the vector of the unknowns, and $\mathbf{b}$ the right-hand side. These are solved using either direct or iterative methods. The most reliable solvers are based on direct methods. The robustness of direct solvers comes at the expense of large storage requirements and execution times. Iterative techniques exhibit problem-specific performance and lack the generality, predictability and reliability of direct solvers. These disadvantages are outweighed by their low memory requirements and their speed especially in the solution of very large matrices.

In the TOUGH2 general-purpose reservoir simulator [Pruess, 1991] the matrix A is a Jacobian with certain consistent characteristics. A has a known block structure with well defined sparsity patterns. Typically, $\mathbf{A}$ is non-symmetric, not positive definite, not diagonally dominant and ill-conditioned. Due to the fact that $\mathbf{A}$ is a Jacobian, the elements of $\mathbf{A}$ in a single row may vary by many orders of magnitude. TOUGH2 creates very challenging matrices with all the attributes that cause most iterative techniques to fail. In addition, the general-purpose nature of TOUGH 2 means that different matrix characteristics may arise for different types of problems. This explains the past heavy reliance of TOUGH2 on the direct solver MA28 [Duff, 19771.

In the current TOUGH2 version, T2CG1, a package of preconditioned conjugate gradient solvers complement the MA28 direct solver and significantly increases the size of tractable problems. T2CG1 includes three solvers: (a) DSLUBC, a routine based on the Bi-Conjugate Gradient (BiCG) method, (b) DSLUCS, a Conjugate Gradient Squared (CGS) routine, and (c) DSLUGM, a Generalized Minimum Residual (GMRES) routine. Tests of T2CG1 [Moridis and Pruess, 1995] on a variety of computing platforms and from systems with up to 30,000 equations have shown that the PCG routines in T2CG1 are significantly (and invariably) faster than MA28 and require far less memory.

In this paper we discuss T2SOLV, an enhanced package of solvers for the TOUGH2 family of codes. It was developed as a replacement for T2CG1, the current solver package. T2SOLV includes all the Preconditioned Conjugate Gradient (PCG) solvers used in T2CG1 as well as a new routine, DLUSTB, based on the Bi-Conjugate Gradient Stabilized (BiCGSTAB) method. T2SOLV also replaces the MA28 by LUBAND, a general banded-matrix direct solver. Additionally, it includes an option for using the D4 ordering scheme and two sets of matrix preprocessors to enhance the PCG performance.

\section{THE LUBAND SOLVER}

LUBAND is a direct solver which replaces the MA28 solver currently used in the TOUGH2 family of codes. It is derived from routines in the LAPACK [1993] package, which have been enhanced and extensively modified to conform to the TOUGH 2 architecture and memory management approach. It is based on a LU decomposition with partial pivoting and row interchange, and allows the solution of systems with a large number of zeros on the main diagonal. Unlike MA28 (which is a general solver), LUBAND is a 
banded matrix solver, and as such it capitalizes on the significantly lower and well defined memory requirements of this class of solvers.

LUBAND can be applied without any problem in the current TOUGH2 version and is fully backward compatible with all older input data files. The MESHMAKER routine was also enhanced to minimize the bandwidth of matrix A. Defining work W as the number of multiplications and divisions necessary to convert the full matrix to an upper triangular form and to perform back substitution, Price and Coats [1974] showed that for direct solvers $W=N M^{2}$ and the minimum storage $\mathrm{S}=N \boldsymbol{B}$, where $\mathrm{N}$ is the order of the matrix and $M$ its half-bandwidth, the full bandwidth being $B=2 M+1$.

For a given problem size $\mathrm{N}$, work and storage are minimized when $\boldsymbol{M}$ is minimized. If $I, J, K$ are the number of subdivisions in the $\boldsymbol{x}$-, $\boldsymbol{y}$ - and $z$-directions respectively, the shortest half-bandwidth is $M=J K$ when $I>J>K$. This is called standard ordering [Aziz and Settari, 19791, and the resulting matrices are banded. As $W$ increases with the square of $\boldsymbol{M}$, it is obvious that the penalty for non-optimization of the ordering of equations may be substantial.

\section{THE DLUSTB SOLVER}

DLUSTB was developed based on the $\operatorname{BiCGSTAB}(m)$ algorithm [Sleijpen and Fokkema, 19931, a recent extension of the more traditional BiCGSTAB algorithm of van der Vorst [1992] which is still an option in T2SOLV. It was developed to address the problem of irregular convergence behavior of the PCG solvers in situations where the iterations are started close to the solution (e.g. when approaching steady state). This is a weakness which afflicts most PCG solvers, and may lead to severe residual cancellation and errors. $\operatorname{BiCGSTAB}(m)$ alleviates the irregular (oscillatory) convergence common to the BiCG [Fletcher, 19761 and CGS [Sonneveld, 19891 methods, thus improving the speed of convergence. It also alleviates potential stagnation or even breakdown problems which may be encountered in traditional BiCGSTAB. According to Sleijpen and Fokkema [1993], BiCGSTAB $(m)$ combines the speed of BiCG with the monotonic residual reduction in the Generalized Minimum Residual (GMRES) method, while being faster than both. Theoretical analysis indicates that the $\operatorname{BiCGSTAB}(m)$ algorithm is especially well-suited to the solution of very large (i.e. $N>50,000)$ problems [vander Vorst, 19921.

DLUSTB uses the Boeing-Harwell matrix storage scheme of TOUGH2, and has the same architecture as the other routines in T2SOLV. As in all other PCG solvers in T2SOLV, it uses a modified LU decomposition for preconditioning. Its memory requirements increase linearly with the order $m$ of the Minimal Residual polynomial. For $\mathrm{m}=4$, it requires twice the memory of BiCG or CGS. The optimum value of $\mathrm{m}$ is calculated internally in DLUSTB.

\section{THE D4 SCHEME}

The Alternating Diagonal Scheme (D4) for gridblock ordering was added as an option to T2SOLV. D4 is a technique belonging to the matrix-banding class of solvers, which derives its benefits from the numbering of the grid points. More details can be found in Price and Coats [1974].

D4 ordering partitions the matrix into four distinct entities. This structure allows forward elimination through the equations in the lower half of $\mathbf{A}$, which zeroes all original entries in the lower left quadrant of A and transforms it into a null matrix, while creating non-zero entries in the submatrix $\mathbf{A}_{L U}$ in the lower right quadrant of $\mathbf{A}$. The submatrix $\mathbf{A}_{L U}$ is of order $N / 2$, and allows the calculation of the lower half of $\mathbf{x}_{\text {, }}$ from which the upper half is obtained by simple substitution. The resulting reduced matrix $\mathbf{A}_{L U}$ can be solved using either direct or iterative methods.

D4 numbering reduces the order of the matrix by $50 \%$ while not increasing the bandwidth. Depending on the grid geometry, D4 makes possible execution speed improvement by a factor ranging between 2 and 5.85 [Price and Coats, 19741 over standard ordering. Moreover, it reduces storage requirements by a factor of 2. Compared to iterative solvers, D4 is competitive in 2-D systems and slower in 3-D systems, and offers the advantage of a robust solution. D4 with LUBAND makes possible the robust direct solution of large multi-dimensional problems. However, D4 can only be used with regular grids.

\section{THE Z-PREPROCESSORS}

Some of the most numerically challenging matrices arising in TOUGH2 simulations involve a large number of zero entries on the main diagonal of the Jacobian. Such matrices are quite common in nonisothermal two-component systems (such as modeling of two-water geothermal systems using the EOS 1 module) and result in at least $0.5 \mathrm{~N}$ of non-zero entries on the main diagonal of the matrix.

Such matrices pose no problem for the LUBAND direct solver. The iterative solvers, however, are directly affected by the diagonal dominance of the matrix and the relative number of the zero entries on the main diagonals. Up to $0.1 \mathrm{~N}$ zero elements have little discernible effect on the PCG solvers in T2SOLV. Matrices with as many as $0.3 \mathrm{~N}$ (and occasionally up to $0.5 N$ ) zero elements are tractable without any special treatment, but usually require a large number of iterations for convergence, i.e. exceeding $0.5 \mathrm{~N}$. 
'he four Z-preprocessors implemented in T2SOLV nhance the performance of the PCG solvers in matries with a large number of main-diagonal zeros. 'hese preprocessors are invoked only when (a) PCG olvers are used to solve (b) matrices with main diaginals populated with a large number of zeros and (c) he number of the primary variables NEQ $>1$.

The first option, Z1, replaces the zeros with a small lumber (typically $10^{-25}$ ), and can substantially derease the number of iterations for convergence in marices with as many as $0.5 \mathrm{~N}$ zero main-diagonal elenents. The performance of the PCG solvers in $\mathrm{Z1}$ rocessed matrices deteriorates rapidly when the mainliagonal zero elements exceed $0.5 \mathrm{~N}$.

The second pre-processing option, 22 , is more comutationally intensive and involves linear combinaions of the flow equations in each gridblock. $\mathrm{Z} 2$ in:ludes a search algorithm which identifies the approJriate equation to be added to the equation correspondng to the zero main-diagonal element. By adding the wo equations, the corresponding elements in the lacobian are replaced with the non-zero sum of the sriginal elements. The 22 option requires limited computational effort and significantly improves the jerformance of the PCG solvers.

While very effective, Z2-preprocessing can still suffer rom poor conditioning because of persisting lack of liagonal dominance and large differences in the magiitude of the added elements. The problem can someimes be alleviated by the 23 option, which precedes :he linear combination with normalization with respect to the largest element in the corresponding row. Addition of the normalized elements leads to an imroved PCG performance because the relative magni:ude of the elements and the corresponding roundoff zrror can be reduced. The 23 option is more computationally intensive than 22 . The 22 and $\mathrm{Z} 3$ preprozessors can easily handle up to $0.75 \mathrm{~N}$ zero diagonal zlements.

I'he 24 pre-processing option is somewhat more zomputationally intensive than 23 . It creates unit main-diagonal submatrices through multiplication by the inverse matrix $\mathbf{A}_{M}{ }^{-1}$, computed using the method of determinants. The PCG performance improvement delivered by $Z A$ can be affected by roundoff errors; under favorable conditions, it matches those of $\mathrm{Z} 2$ and Z3.

\section{THE O-PREPROCESSORS}

The O-preprocessors are applied to matrices with no zero entries on the main diagonal and aim to improve the PCG solver performance by improving the matrix conditioning. Four such preprocessors are available in T2SOLV. The first three options, 01 through 03 , are in essence steps in the replacement of the $\mathbf{A}_{M}$ submatrix by the unit matrix through a central pivoting process, and involve increasing levels of computational effort.

The 01 option eliminates the lower half of the maindiagonal submatrix, and thus removes NEQ- 1 subdiagonals from the global matrix. This reduces the computational effort by reducing the number of nonzero matrix entries and can improve the PCG performance. Execution times are burdened by the additional work for the elimination of the lower half of the matrix, but usually this is overcome by the savings in the PCG computations.

In the 02 option, in addition to 01 the upper half of the main-diagonal submatrix is eliminated, resulting in a diagonal submatrix and eliminating an additional NEQ- 1 superdiagonals from the global matrix. Compared to the original, the $\mathrm{O} 2$-preprocessed matrix is significantly sparser and better-conditioned and the performance of the PCG solvers can be enhanced. The increased computational effort for the 02 preprocessing is usually compensated by the reduction in the PCG iterations.

The 03 options involves normalization of the 02 matrix, resulting in a unity main diagonal. 03 does not further increase matrix sparsity, but may improve the matrix conditioning. Finally, the 04 option is identical to the $Z A$ option discussed previously.

\section{TEST PROBLEMS}

The solvers were tested in four test problems. Performance results are presented in Figures 1 through 3 and Tables 1 through 4 .

\section{Test Problem 1}

Test problem 1 involves a study of non-isothermal flow in a "two-water" system. Such systems are known to be the most challenging for the solvers in TOUGH2, as they routinely create matrices with $0.67 \mathrm{~N}$ zeros on the main diagonal. The PCG routines in T2CG1 have in the past been unable to solve even the smallest of this class of problems. The problem discussed here involves injection of "water $2 \mathrm{n}$ at a temperature of $30^{\circ} \mathrm{C}$ into a geothermal reservoir of "water $1 "$ at $280^{\circ} \mathrm{C}$. The EOSl module is used. The $3-D$ domain consists of $9 \times 8 \times 5=360$ gridblocks in $(x, y, z)$, with $\mathrm{NK}=2$ and NEQ $=3$, resulting in a total of $\mathrm{N}=1080$ equations.

The fundamental weakness of MA28, i.e. its large (especially for 3-D problems) and not well defined memory requirement, was obvious in the problem. Despite memory allocation which sufficed for the LUBAND solution of $3-D$ problems 15 times larger, MA28 could not complete the LU decomposition due to insufficient memory. 
Table 1 and Figure 1 show that DLUSTB with and without the Z-preprocessors has the best performance. It is the fastest and requires the least number of PCG iterations to convergence. DLUSTB seems to be the only solver that can proceed without Z-preprocessing. Note that the use of the Z-preprocessors makes possiole the solution of a previously-intractable problem oy all the PCG solvers in T2SOLV. The Z2 preprozessor seems to offer the best overall performance.

\section{rest Problem 2}

rest problem 2 involves a laboratory convection cell experiment. A porous medium consisting of glass beads fills the annular region between the two vertical zoncentric cylinders. Application of heat generates a thermal buoyancy force, giving rise to the development of convection cells. This problem has been diszussed in detail by Moridis and Pruess [1992]. The EOS1 module is used. The domain consists of $16 \times 26=416$ gridblocks in $(r, z)$, with $\mathrm{NK}=1$ and $\mathrm{NEQ}=2$, resulting in a total of $\mathrm{N}=832$ equations.

Table 2 and Figures 2 and 3 show the performance of the various solvers in Problem 2, which does not pose any significant challenges to the T2SOLV routines. DLUSTB is the fastest routine and requires the least number of iterations to convergence.

In this 2D problem LUBAND appears as a competitive alternative. The effect of the 0 -preprocessors vary. With 01 , it is pronounced in terms of PCG iterations and execution times in DSLUBC and DLUSTB, but seems to be limited in DSLUCS and DSLUGM. The evolution of residuals of DSLUCS and DSLUGM in the first Newtonian iteration of the first timestep is identical with and without 01 preprocessing (Figures 2 and 3), while the DSLUCS execution time with 01 increases. Conversely, the use of the 02 and 03 preprocessors seems to offer the greatest improvement in the performance of DSLUCS and DSLUGM.

\section{Test Problem 3}

Test problem 3 examines fluid and mass flow in a large three-dimensional model of a geothermal reservoir. The basic computational grid is composed of $15 \times 15 \times 20=4500$ grid blocks in $(x, y, z)$. Cold water is injected through 4 wells, while hot water is withdrawn from 5 wells. EOS1 is used with $\mathrm{NK}=1$, NEQ $=2$, resulting in a total of $N=9000$ equations.

This is a relatively large but well-behaved problem, the size of which precluded the use of a direct solver. The use of $\mathrm{D} 4$ allowed a direct solution by LUBAND, which is competitive with the PCG solutions. D4 with DLUSTB had a performance on a par with DLUSTB, the fastest PCG solver. In light of the overhaed needed to set up the D4 system, this result is very encouraging.
DLUSTB demonstrated its superiority by being the fastest and requiring the least number of PCG iterations to convergence. DSLUGM seems to be an inappropriate method for this type of problem. As expected, the benefits of 0 -preprocessing in this wellbehaved system are not evident in the execution times, although the PCG iterations are often reduced. It is noteworthy, however, that despite the increased computational load, the execution times for the $\mathrm{O}$ preprocessed solutions are practically identical to those without any preprocessing.

\section{Test Problem 4}

Test problem 4 describes a variation of the Thermal Enhanced Vapor Extraction System process, which is designed to extract solvents and chemicals contained in the Chemical Waste Landfill at Sandia National Laboratories. No NAPL is present in this system. In this process the ground is electrically heated, and boreholes at the center of the heated zone are maintained at a vacuum to draw air and vaporized contaminants into the borehole and to a subsequent treatment facility. The 3-D grid consists of 1300 gridblocks. EOS3 is used (NK $=2, \mathrm{NEQ}=3$ ), and $\mathrm{N}=3900$ equations are solved. Additional information can be found in Moridis and Pruess [1995].

Without any preprocessing, the performance of DLUSTB in this rather well-behaved problem is practically identical to that of DSLUCS. These two are the fastest solvers, but DLUSTB requires the least number of PCG iterations. D4 with LUBAND appears as a competitive alternative. DLUSTB is the most responsive to 01 preprocessing, which results in the fastest solution with the least number of PCG iterations.

\section{CONCLUSIONS}

The following conclusions can be drawn:

(1) Without any matrix preprocessing, DLUSTB is shown to be a fast and efficient solver which outperforms the other PCG routines. It is the fastest and the most robust in T2SOLV and is shown to be practically free of stagnation, oscillation, and divergence problems.

(2) The use of the Z-preprocessors makes possible the solution of problems which were previously intractable to all the PCG solvers. The combination of the Z-preprocessors with the BiCGSTAB routine gives the best performance in such problems.

(3) In problems which are known to confound the other PCG solvers, DLUSTB converges smoothly but slowly to a solution without invoking the matrix-preprocessing facility. 
(4) The 0-preprocessors are shown to improve the robustness and decrease the number of iterations to convergence, but their effect depends on the PCG solver in T2SOLV. DLUSTB appears to be the solver most consistently responsive to the 0 -preprocessors. In well-behaved problems the effect of the O-preprocessors on the execution speed is not significant.

(5) LUBAND is shown to be consistently faster and more reliable than MA28, and can solve much larger problems.

(6) The gains in execution speed when the D4 scheme is used in regular grids are shown to be significant (especially compared to the direct solver). D4 with direct matrix solution seems to be competitive (in speed) to the PCG solvers in medium-sized problems, although this advantage is expected to disappear in large problems due to memory limitations.

Table 1. Solver Performance in Problem 1 (Macintosh PowerPC 9500/132)

\begin{tabular}{|c|c|c|c|c|c|c|c|}
\hline SOLVER & $\mathbf{P P}$ & $\Delta t s$ & $\mathbf{N I}$ & $\operatorname{lm} x$ & Imn & IT & ET \\
\hline MA28 & \multicolumn{7}{|c|}{ Fails - insufficient memory } \\
\hline LUBAND & - & 8 & 25 & - & - & - & 63.9 \\
\hline \multirow[t]{4}{*}{ DSLUBC } & - & \multicolumn{6}{|c|}{ Fails } \\
\hline & $\mathrm{Z1}$ & \multicolumn{6}{|c|}{ Fails } \\
\hline & 22 & 8 & 29 & 109 & 5 & 890 & 29.0 \\
\hline & $\mathrm{Z3}$ & 8 & 28 & 109 & 7 & 877 & 29.6 \\
\hline \multirow[t]{4}{*}{ DSLUCS } & - & \multicolumn{6}{|c|}{ Fails } \\
\hline & $\mathrm{Z1}$ & \multicolumn{6}{|c|}{ Fails } \\
\hline & 22 & 12 & 41 & 109 & 4 & 1002 & 38.6 \\
\hline & 23 & 12 & 46 & 100 & 4 & 1253 & 46.5 \\
\hline \multirow[t]{4}{*}{ DSLUGM } & - & \multicolumn{6}{|c|}{ Fails } \\
\hline & $\mathrm{Z1}$ & \multicolumn{6}{|c|}{ Fails } \\
\hline & 22 & 8 & 30 & 51 & 7 & 531 & 21.3 \\
\hline & $\mathrm{Z3}$ & 8 & 28 & 27 & 2 & 496 & 19.3 \\
\hline \multirow[t]{4}{*}{ DLUSTB } & - & \multicolumn{6}{|c|}{ Fails } \\
\hline & $\mathrm{Z1}$ & 15 & 94 & 109 & 7 & 642 & 93.2 \\
\hline & $\mathrm{Z2}$ & 8 & 25 & 20 & 4 & 289 & 16.9 \\
\hline & $\mathrm{Z3}$ & 8 & 25 & 27 & 4 & 288 & 17.0 \\
\hline
\end{tabular}

PP: Preprocessors

Ats: Number of timesteps

NI: Newtonian iterations

Imx: Maximum number of PCG iterations

Imn: Minimum number of PCG iterations

IT: Total PCG iterations

ET: Execution time (sec)

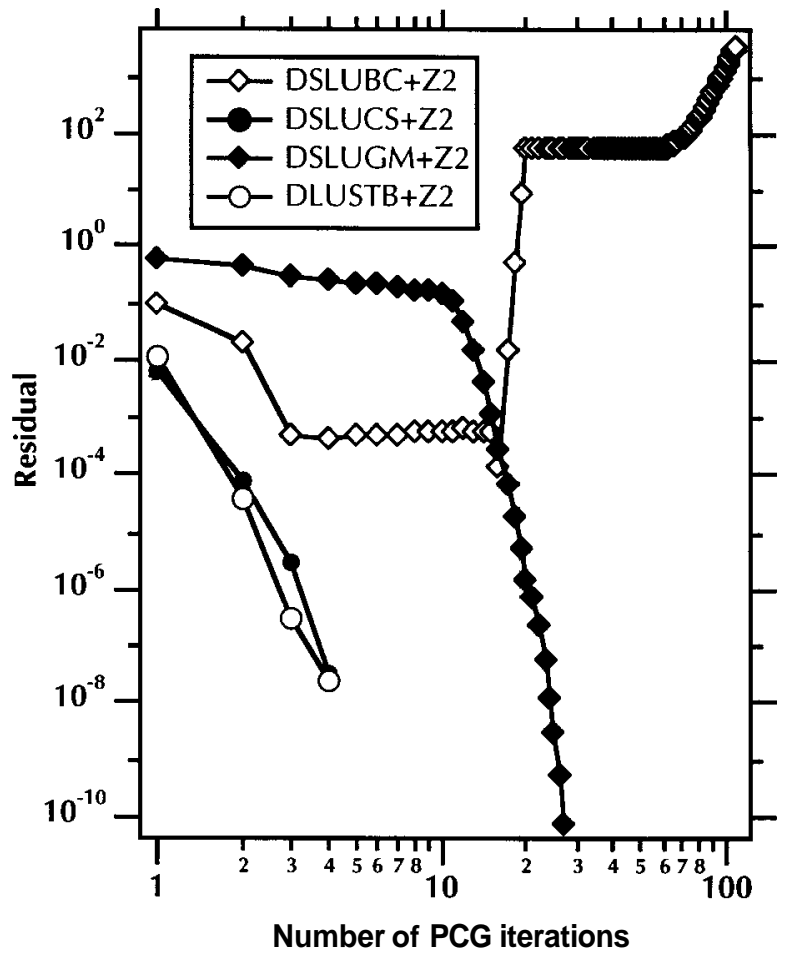

Fig. I. PCG solvers with Z2 preprocessing in Test Problem I (1st NI of the 1st At).

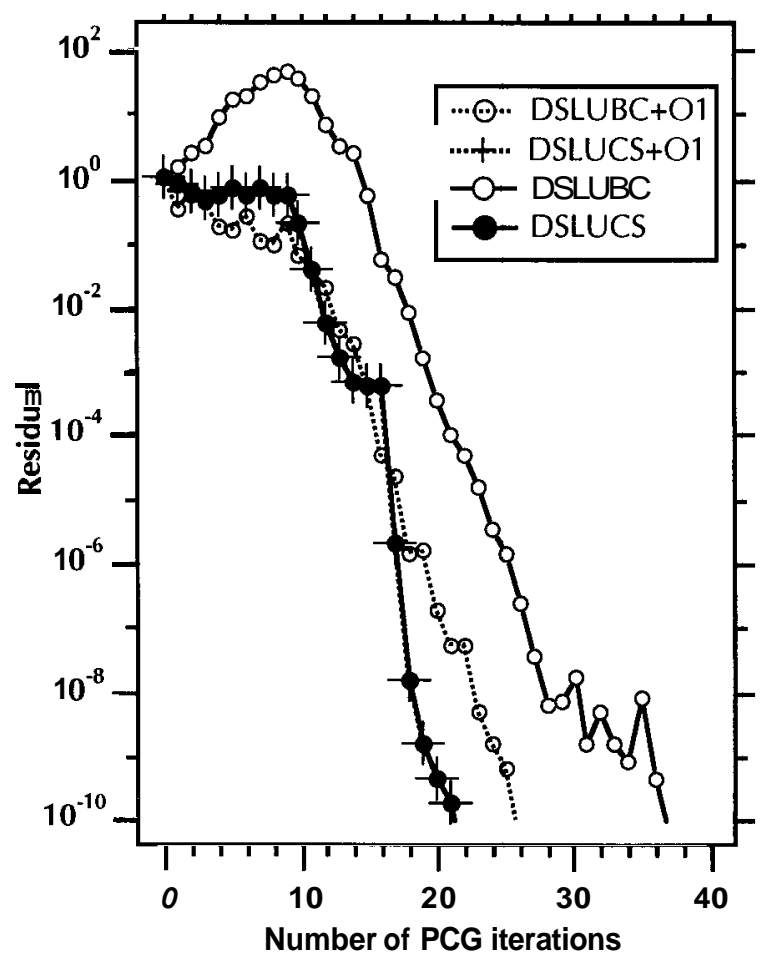

Fig. 2. DSLUBC and DSLUCS performance with and without 01 preprocessing in Test Problem 2 (1st NI of the 1st At). 


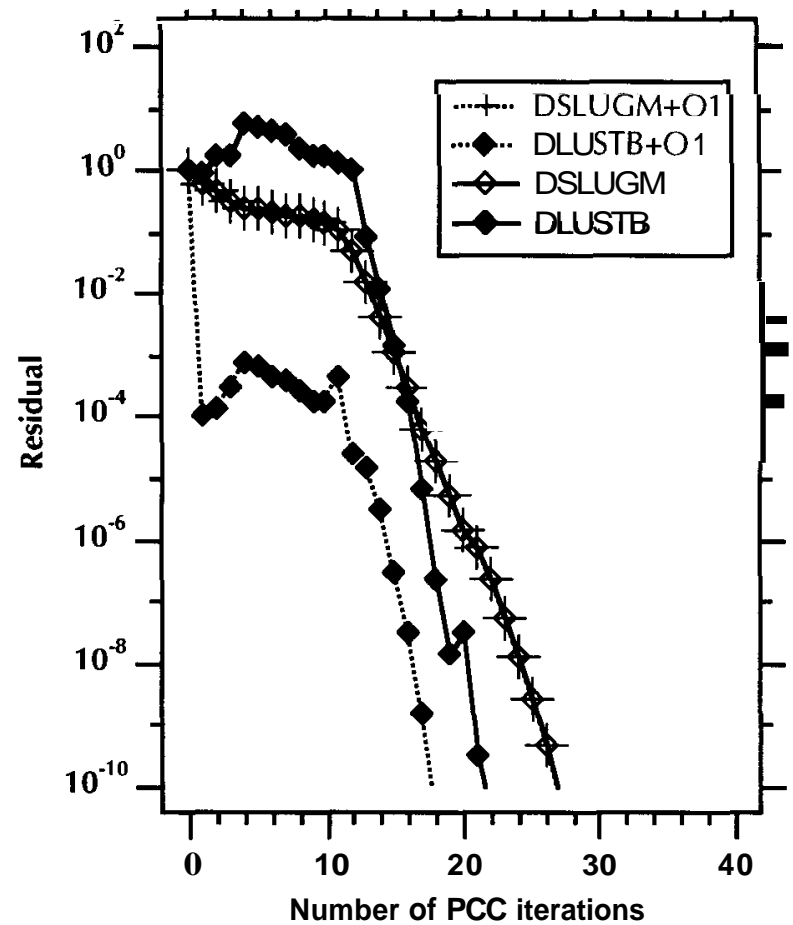

Fig. 3. DSLUGM and DLUSTB performance with and without 01 preprocessing in Test Problem 2 (1st NI of the 1st At).

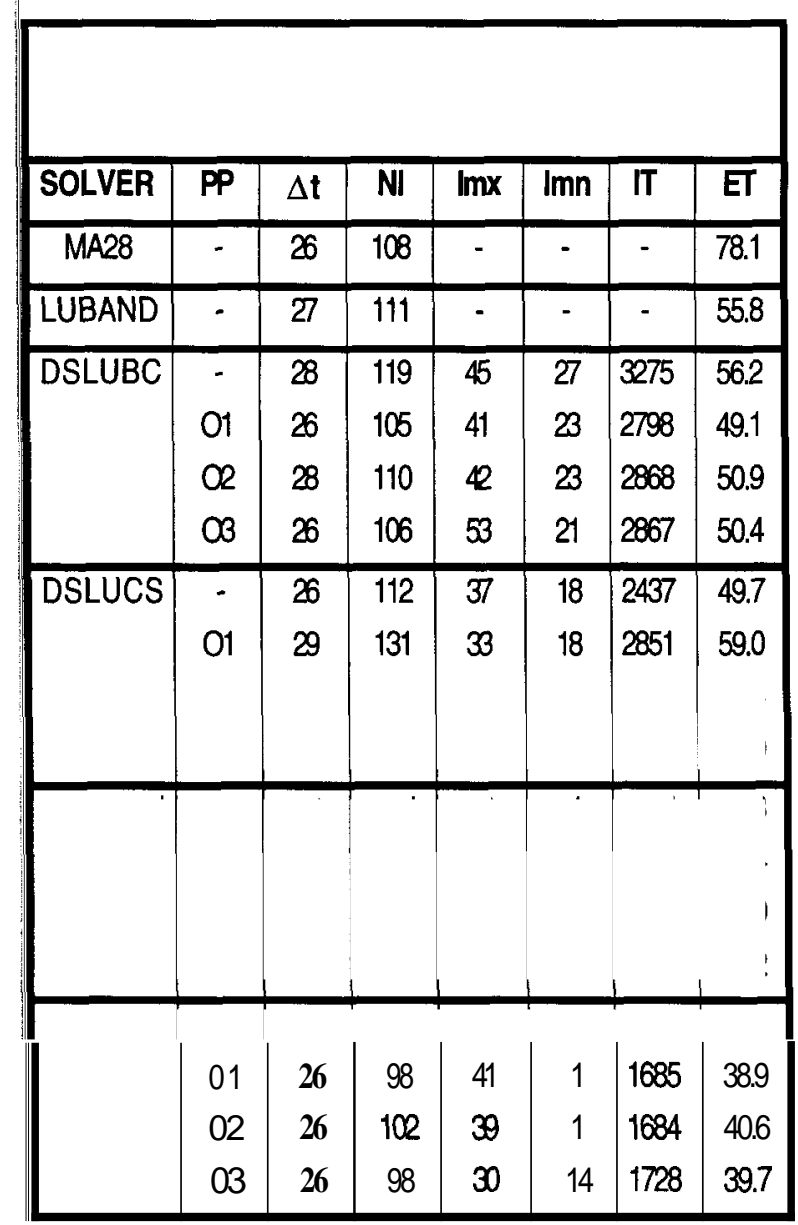

\begin{tabular}{|c|c|c|c|c|c|c|c|}
\hline \multicolumn{7}{|c|}{ Ins } \\
\hline SOLVER & PP & $\Delta t$ & NI & Imx & Imn & IT & IT \\
\hline MA28 & \multicolumn{7}{|c|}{ Insufficient Memory } \\
\hline LUBAND & \multicolumn{7}{|c|}{} \\
\hline D 4t & - & 10 & 46 & - & & - & 786 \\
LUBAND & & & & & & & \\
\hline D44+ & - & 10 & 46 & 57 & 4 & 1736 & 426 \\
DLUSTB & & & & & & & \\
\hline DSLUBC & - & 10 & 46 & 106 & 63 & 2623 & 579 \\
& 01 & 10 & 46 & 75 & 57 & 2477 & 565 \\
& 02 & 10 & 46 & 75 & 52 & 2475 & 563 \\
\hline DSLUCS & - & 10 & 46 & 95 & 50 & 2051 & 488 \\
& 01 & 10 & 46 & 98 & 50 & 2034 & 485 \\
& 02 & 10 & 46 & 94 & 50 & 2033 & 487 \\
\hline JSLUGM & - & 10 & 46 & 930 & 95 & 14842 & 2087 \\
& 01 & 10 & 46 & 930 & 96 & 14994 & 2178 \\
& 02 & 10 & 46 & 930 & 95 & 15113 & 2189 \\
\hline DLUSTB & - & 10 & 46 & 58 & 41 & 1736 & 423 \\
& 01 & 10 & 46 & 60 & 37 & 1695 & 421 \\
& 02 & 10 & 46 & 58 & 37 & 1719 & 429 \\
\hline
\end{tabular}

Table 4. Solver Performance in Problem 4 (DEC AlphaStation 200/233)

\begin{tabular}{|c|c|c|c|c|c|c|c|}
\hline SOLVER & PP & $\Delta t$ & NI & Imx & Imn & IT & ET \\
\hline MA28 & \multicolumn{7}{|c|}{ Insufficient Memory } \\
\hline LUBAND & - & 50 & 249 & - & - & - & 1227 \\
\hline D4+ + & - & 50 & 249 & - & - & - & 678 \\
\hline LUBAND & & 50 & & & & & \\
\hline DSLUBC & - & 50 & 249 & 29 & 7 & 4756 & 712 \\
& 01 & 50 & 250 & 28 & 7 & 4712 & 701 \\
\hline DSLUCS & - & 50 & 249 & 29 & 4 & 3681 & 639 \\
& 01 & 50 & 249 & 26 & 4 & 3669 & 649 \\
\hline DSLUGM & - & 50 & 249 & 27 & 7 & 3986 & 506 \\
& 01 & 50 & 249 & 26 & 7 & 3984 & 508 \\
\hline DLUSTB & - & 50 & 249 & 15 & 4 & 2544 & 509 \\
& 01 & 50 & 249 & 15 & 4 & 2232 & 484 \\
\hline
\end{tabular}




\section{ACKNOWLEDGMENT}

This work was supported by the Assistant Secretary for Energy Efficiency and Renewable Energy, Office of Geothermal technologies, of the U.S. Department of Energy, under contract No. DE-AC03-76SF00098. Drs. Curt Oldenburg and Stefan Finsterle are thanked for their helpful review comments.

\section{REFERENCES}

Aziz, K. and A. Settari (1979), Petroleum Reservoir Simulation, Elsevier, London and New York.

Duff, I.S. (1977) MA28 - A set of Fortran Subroutines for Sparse Unsymmetric Linear Equations, AERE Harwell Report R 8730.

Fletcher, R. (1976), Conjugate gradient methods for indefinite systems. Numerical Analysis, Lecture Notes in Mathematics 506, Springer-Verlag, New York.

LAPACK (1993), Univ. of Tennessee, Univ. of California at Berkeley, NAG ltd., Courant Institute, Argonne National Lab., and Rice University, Version 1.1 .

Moridis, G.J. and K. Pruess (1992), TOUGH simulations of Updegraffs set of fluid and heat flow problem, Lawrence Berkeley Laboratory report LBL32611, Berkeley, CA.

Moridis, G.J. and K. Pruess (1995), T2CG1: A package of preconditioned conjugate gradient solvers for the TOUGH2 family of codes, Lawrence Berkeley Laboratory report LBL-36235, Berkeley, CA.

Price, H. S. and K. H. Coats (1974), Direct methods in reservoir simulation, Trans. SPE of AIME (SPEJ), 257, 295-308.

Pruess, K (1991), TOUGH2 - A general-purpose numerical simulator for multiphase fluid and heat flow, Lawrence Berkeley Laboratory report LBL29400, Berkeley, CA.

Sleijpen, G.L.G. and D. Fokkema (1993), BiCGSTAB(1) for linear equations involving unsymmetric matrices with complex spectrum, Electronic Transactions on Numerical Analysis, 1, 11-32.

Sonneveld, P. (1989), CGS, A fast Lanczos-type solver for nonsymmetric linear systems. SIAM J. Sci. Stat. Comput., 10(1), 36-52.

van der Vorst, H.A. (1992), Bi-CGSTAB: A fast and smoothly converging variant of Bi-CG in the presence of rounding errors, SIAM J. Sci. Statist. Comput., 13, 631-644. 